\title{
Ejecución del programa "Evaluación por competencias" implementado por el Ministerio de Educación Pública en la Educación Técnica: el caso del Colegio Técnico Profesional de San Sebastián
}

\author{
Execution of the "competency assessment" program implemented by the Ministry of Public Education in the \\ Technical Education: the case of Professional Technical School in San Sebastian
}

Recibido 29 octubre 2013 • Aceptado 02 diciembre 2013 • Corregido 11 diciembre 2013

\author{
Jorge Retana Morales ${ }^{1}$ \\ Ministerio de Educación Pública \\ San José, Costa Rica \\ alo2178@gmail.com
}

\begin{abstract}
Resumen. Este artículo refiere los resultados de la investigación realizada sobre el programa Evaluación por Competencias, implementado por el Ministerio de Educación Pública en la Educación Técnica en el Colegio Técnico Profesional de San Sebastián, a partir del diagnóstico que tienen los educadores sobre la propuesta, la identificación de dificultades y ventajas en su aplicación. El estudio es de carácter cualitativo con un enfoque descriptivo, en el que participan docentes y directivos a los que se les realiza entrevistas para obtener la información más precisa de la realidad del programa en ese centro educativo. Entre los hallazgos se manifiestan debilidades en la ejecución del programa, debido al desconocimiento de conceptos básicos y lineamientos del programa de Evaluación por Competencias. Como ventajas, se determina el acercamiento de la evaluación al mundo laboral en que se va a desempeñar el estudiante, a partir de sus competencias específicas, por lo que se resalta la importancia de la administración de la educación no formal para gestar procesos socioeducativos en ese sentido.
\end{abstract}

Palabras claves. Educación, evaluación por competencias, administración de la educación no formal

Abstract. This article refers the results of the research about the program assessment by competences, implemented by the Ministry of public education in the technical education in the Professional Technical School in San Sebastian, from diagnosis the educators have on the proposal, the identification of difficulties and advantages in your application. The study is qualitative with a descriptive approach, involving teachers and managers to which are performed interviews to obtain the most accurate information of the reality of the program at that school. Among the findings is demonstrated weakness in the implementation of the program, due to the lack of knowledge of basic concepts and guidelines of the competency assessment program. As advantages, is determined the evaluation approach to the world of work, which will play the student, from their

1 Licenciado en Ciencias de la Educación con énfasis en Administración de la Educación No Formal de la Universidad de Costa Rica (UCR). Licenciatura en Docencia de la Universidad de San Marcos (USAM). Bachillerato en la Enseñanza del Inglés de la Universidad Nacional (UNA). Se ha desempeñado profesionalmente en el Instituto Tecnológico de Costa Rica (ITEC) y el Ministerio de Educación Pública (MEP). 
specific competences, so that the importance of non-formal education management stands out to gestate processes socio-educational in that sense.

Keywords. Education, competence assessment, administration of non-formal education

\section{Contextualización}

El siglo XX se caracterizó por una serie de cambios vertiginosos incidiendo en la sociedad en general, como los avances tecnológicos sin precedentes, la desintegración de estructuras de poder, la ruptura de paradigmas rígidos y obsoletos, lo que contribuye a nuevas formas de percibir el mundo, las representaciones y normas de socialización. Estos cambios, el desarrollo de tecnologías e innovaciones, han transformado la manera de relacionarse en los distintos campos sociales y en los diferentes campos del saber.

Estas variaciones se dieron en todos los niveles de las ciencias y la educación, durante el siglo XX; producto de ello, se han ido modificando los paradigmas de aprendizaje de la educación. Nuevas corrientes y metodologías han surgido con el fin de hacer un mejor manejo de todos los recursos que el ser humano tiene a su disposición y así, enriquecer todos sus procesos en la vida cotidiana, como en lo educativo, científico y laboral, entre otros. La globalización ha impactado en la sociedad, de manera que todo fenómeno social se interrelaciona, en forma directa o indirecta, a la dinámica global; en consecuencia, los acontecimientos económicos, políticos y sociales se asemejan cada vez más a la aldea global.

Se ha llegado al siglo XXI con grandes avances en todas las áreas de las ciencias y los saberes, pero también, con grandes deficiencias en el plano humano y social. Las nuevas corrientes e ideas dentro de la enseñanza y la educación, buscan un ser humano más sistémico, atento a todo aquello que le rodea y además, pueda cumplir un rol de productividad, que es el que la sociedad demanda. De acuerdo con Vargas (1998), estos efectos no excluyen a ningún contexto:

Muchas de las actuales tendencias atestiguadas en el mundo, no excluyen de sus efectos prácticamente a ningún país. La búsqueda de mejores niveles de bienestar y de un mayor grado de desarrollo es ahora un factor en el que coinciden los países firmantes de tratados de libre comercio y de creación de áreas comunes y uniones como (el tratado de libre comercio de América del norte TLCAN, el mercado común del sur MERCOSUR, la comunidad del Caribe CARICOM y la Unión Europea (UE). (p. 49)

Estos tratados han traído consigo que las corporaciones y empresas se muden de sus países de origen, buscando aquellos países con más estabilidad y desarrollo humano, o con mano de 
obra barata, lo que crea distintas fragmentaciones del trabajo, así como en distinto campos tales como la investigación, modelos de producción y manufactura, entre otros. Conduciendo esto, a que la producción de un solo artículo y sus procesos de fabricación sean llevados a cabo en una variedad de naciones, dependiendo de la estabilidad del país, su costo, sus recursos naturales y humanos.

Es una necesidad social el invertir en su capital humano, producto de las transformaciones en los campos tecnológicos, de las ciencias, la educación y la medicina, entre otros, ya que estos cambios surgen cada vez más rápido, al modificar el mundo y su contexto social. Al respecto, Vargas (1998, p. 50) considera que "(...) la ubicación que cada país tenga en la nueva división internacional va a depender mucho de su dotación de talento humano, y ya no de sus recursos naturales o de su riqueza material". Las riquezas materiales han traído consigo ganancias a ciertos países y zonas, por lo general las más industrializadas, que han tenido a lo largo del siglo pasado altos índices de inversión en investigación y educación. De esta forma, las naciones que quieran alcanzar el desarrollo deben buscar apropiarse de la información, así como crear nuevos conocimientos en beneficio de sus sociedades.

Los cambios que se han dado a lo largo del siglo XX y que se siguen acentuando a inicios del siglo XXI, han aumentado las políticas de exclusión social y la generalización del desempleo y la pobreza. En este siglo y el anterior, se han profundizado las inequidades en cuanto al desarrollo social, económico y tecnológico y a la distribución de la riqueza entre los países, lo que trae consigo grandes diferencias en la calidad de vida de los habitantes de las distintas regiones del mundo. En los últimos años del siglo XX y comienzos del siglo XXI, se ha consolidado el fenómeno de la globalización, el cual ha hecho más notoria la diferencia o brecha educacional y social en el plano digital y tecnológico, debido a que la información está en manos de unos pocos que pueden tener acceso a ella. El conocimiento es el soporte para la información, de modo que se ha convertido junto con la educación, en la base para la generación de riqueza y prosperidad.

Los acontecimientos del siglo XX y los que empiezan en el siglo XXI, han incidido en la estructuras más importantes de las sociedad occidental y permean en el resto del planeta; así la educación, sus instituciones, el trabajo, el contexto social y económico de las masas populares han sido obligadas a transformarse ante las diferentes fuerzas de los actores sociales que existen en un mundo cada vez más inmediato y pequeño, en el que surgen más conocimientos e informaciones. La educación se ha transformado debido a la invención de dispositivos o aditamentos tecnológicos que se centran y mejoran la información. Estas transformaciones influyen en todos los actores y están asociados al avance de las tecnologías de manejo, procesamiento, acceso y distribución de información. Al respecto, Vargas (1998, p. 50) considera que «(...) no han pasado aún 60 años después de la irrupción del computador y se afirma ya que en materia de información se avanzó más en los años transcurridos luego de la segunda guerra mundial, que en toda la evolución humana desde el paleolítico hasta la fecha". 
Entre los cambios que han generado las tecnologías en los diferentes ámbitos de la vida del hombre, se puede nombrar la masificación de los productos, los estándares de calidad y su rápida obsolescencia, generando un mercado de consumo sin precedentes. Esto se traduce en una demanda de ocupación más específica y especializada. Las personas, en este contexto, deben de ser capaces de cubrir otras áreas en sus ocupaciones de modo que la flexibilidad y su capacidad de resolver situaciones complejas sea una norma. Las personas que se desempeñan en el campo de servicios deben cumplir una serie de competencias genéricas que hacen del trabajador, una persona valiosa en su área de trabajo, debido a su integridad y altos valores humanos.

Los conocimientos, la educación, el trabajo y la forma de competir han cambiado sus métodos tradicionales y rígidos a unos más flexibles e integrales, en los que toma relevancia una serie de aspectos ligados a la complejidad que caracteriza el mundo y sus relaciones con el medio. Para responder en forma acertada y competente a los cambios emergentes que inciden en las demandas laborales, surgen enfoques orientados a la calificación laboral. Estos enfoques van dirigidos a un ser humano que posea diferentes cualidades que están ligadas a su historia de vida y lo conviertan en una persona íntegra, capaz de lidiar con diferentes tareas y cuyo sentido del medio que le rodea es muy agudo para así, enfrentar los diferentes contextos que el ambiente complejo de la sociedad contemporánea demanda hoy día.

Además, de estos enfoques, Vargas menciona (1998):

Que hoy en día la formación profesional debe de buscar la generación de competencias en los trabajadores y no la simple conjunción de habilidades, destrezas y conocimientos. La configuración adquirida por las ocupaciones exige a los trabajadores un más amplio rango de capacidades que involucran no sólo conocimientos y habilidades sino también la comprensión de lo que están haciendo. Paulatinamente se piden más competencias de contenido social asociadas a la comunicación, capacidad de diálogo, capacidad de negociación, pensamiento asertivo y facilidad para plantear y resolver problemas. (p. 53)

Para alcanzar la meta que el mercado requiere, en el que las corporaciones, empresas o empleadores solicitan un personal que ocupe, de forma eficiente, una plaza de trabajo en que, además de sus cualidades posea una serie de capacidades que lo permitan desarrollarse y emplearse a fondo en beneficio de los objetivos de trabajo, las corporaciones y las empresas crean binomios con los estados de los países; de esta forma, sus políticas educativas se convierten en lineamientos claros que suplan las demandas que las plazas de trabajo exigen. Los avances sociales han cambiado el escenario laboral, exigiendo una mano de obra cada vez más calificada, según las demandas de los nuevos tiempos. En esta asociación manifiesta, entidad 
pública o empresa privada, el estado se compromete a crear el ambiente propicio dentro de sus instituciones educativas para hacer frente a la demanda ocupacional que se le solicita.

Es debido a los adelantos que el ser humano ha tenido en los diferentes ámbitos y a la modificación de las estructuras sociales, en los últimos años, que se busca armonizar estos cambios con un enfoque que integre todos los cambios que los nuevos retos de la sociedad exige y ligar el sistema educativo de un Estado, con la productividad de éste y la que el mundo demanda. De esta forma, según Ruiz (2003):

El mundo actual se caracteriza por una marcada tendencia hacia la globalización de la economía. Además, los mercados de bienes y servicios son cada vez más competitivos y el acelerado cambio tecnológico impone nuevas competencias laborales a los profesionales. Ello plantea la necesidad de una transformación drástica en las nociones y formas de realizar los aprendizajes, fortaleciendo los lazos con el sector productivo. (p. 81)

Partiendo de esta premisa, surge este nuevo modelo de educación, el cual se adapta a los métodos ya existentes de educación y está acorde con la noción de ser humano imperante en el mundo desarrollado; la formación por competencias no es algo nuevo, como lo menciona Ruiz (2003) incluso países como «Gran Bretaña, España y México, avanzan en la formación de trabajadores para el desempeño productivo con modelos fundamentados en competencias laborales" (p. 83). Las afirmaciones de la competitividad, la globalización de la economía, además del acelerado cambio tecnológico ya mencionado, entre otros, muestran la necesidad que tienen los países de adaptarse a un enfoque laboral más acorde al modelo de empleado que se ocupa hoy día, para el óptimo desarrollo en los diferentes campos y contextos laborales que demandan las sociedades actuales.

La administración de la educación no formal debe, y está llamada a ser, un agente significativo de cambio para afrontar los retos que el mercado laboral demanda hoy día, ya que está aplicada al campo de los procesos socioeducativos, puesto que su ámbito de acción implica diseñar, organizar, ejecutar y evaluar actividades educativas con el propósito de mejorar las condiciones de vida de diversos grupos humanos, mediante procedimientos participativos promotores de transformaciones individuales y colectivas. Las diferentes modalidades de educación no formal pueden ser agentes significativos de cambio para el rezago o actualización de personas que quieren estar a punto con las demandas que la sociedad demanda, pero visualizando a futuro, la administración de la educación no formal, desde sus diferentes mecanismos sean políticos estatales o privados, como organizaciones no gubernamentales, son y pueden ser una herramienta fundamental para los países que deseen implementar proyectos, capacitaciones, talleres, entre otros, que ayuden a apuntalar y mejorar los diferentes 
sistemas educativos de una sociedad y, por lo tanto, adaptar ésta a los cambios tecnológicos y educacionales, en beneficio de toda la nación.

La administración de la educación no formal, según sea un sistema o proceso educativo, tomada como educación externa al sistema formal, de acuerdo con Rogers (2004), puede ser un importante agente creador de cambio en una región o país con planes de adaptarse a los cambios que se están generando en la aldea global de hoy.

La administración de la educación no formal puede generar cambios muy específicos en una población, mediante procesos intencionales de instrucción, sistemáticos y organizados, diseñados para alcanzar objetivos de aprendizaje específicos por un grupo particular de estudiantes, tal como menciona Rogers (2004), dentro del ámbito externo de la educación formal, que provee y selecciona diferentes tipos de aprendizaje a subgrupos particulares de la población como jóvenes y adultos.

La administración de planes educativos de educación no formal, resulta una alternativa práctica y flexible para complementar con la educación formal $y$, de esta forma, plantear programas o proyectos que, bien coordinados entre los dos, puede sacar a relucir lo mejor de cada sistema. Las regiones o países del mundo, por medio de sus administraciones, pueden echar mano de la amplitud y flexibilidad de la educación no formal y crear sistemas, colección de organizaciones o programas del mismo sistema de educación no formal, contextualizados como un proceso de diferentes relaciones de enseñanza aprendizaje.

La amplitud que ofrecen los programas de educación no formal a la administración de la educación es muy extensa e inclusiva, ya que sus alcances son para todos los actores sociales, desde niños, adultos, jóvenes en instituciones formales, adultos fuera de los ámbitos formales de educación o jóvenes con rezagos educativos, entre otros. Los nuevos paradigmas que plantea el mundo laboral y educacional son propicios para que, desde la administración educativa y los programas o proyectos de educación no formal, más personas puedan acceder a las nuevas herramientas tecnológicas y de conocimiento, así como tener la posibilidad de nuevas aspiraciones y actitudes renovadoras que impulsen a las personas a su desarrollo personal. Desde la administración de la educación no formal, se pueden resolver problemas de la educación formal y viceversa. Entre otras ventajas que puede ofrecer la educación no formal desde la implementación de planes de la administración de la educación y que puedan impulsar o catapultar el desarrollo de un país están, a criterio de Rogers (2004):

- La educación no formal busca formas alternativas de educación que sean más efectivas en la consecución de las metas.

- La educación no formal se identifica con todos aquellos procesos de socialización que brindan herramientas de aprendizaje llevados a cabo fuera de la educación formal. 
- Esta intencionada para que por medio de programas o capacitaciones separadas o complementarias a la educación formal se desarrollen destrezas en adultos o jóvenes como por ejemplo, comunicación, mejorar actitudes o modificar comportamientos relacionados a la consecución de metas de desarrollo y alcanzar más altos estándares de vida y de bienestar.

- La educación no formal es sinónimo de clases cortas, orientadas a actividades y problemáticas sociales y políticas.

- Puede ser tomada como una educación básica y para adultos como la vocacional pero fuera del sistema escolar.

- Es barata puesto que puede usar la infraestructura existente de la educación formal

Los procesos de la educación no formal son administrados de modo que se planifiquen el conjunto de actividades y acciones de forma articulada, de manera que, previstas anticipadamente, tengan el propósito de influir en el curso de determinados acontecimientos, con el fin de alcanzar una situación elegida como deseable, mediante el uso eficiente de medios y recursos escasos o limitados; la administración de la educación no formal puede convertirse en un mecanismo efectivo y significativo para que la sociedad costarricense dé el salto que necesita para alcanzar, en este caso, la formación basada en competencias para la mejora de la sociedad.

La adaptación de los docentes al enfoque de formación por competencias no ha sido fácil; se ha trabajado por medio del Departamento de Educación Técnica mediante la breve inducción de una charla de lo que es el enfoque por competencias laborales. Es necesario, para hacer el proceso de enseñanza aprendizaje más eficaz, integrar el enfoque basado en competencias. Este enfoque está en concordancia con el panorama imperante en el mundo en el que se persigue, según Segura (2009, p. 10), una noción «integral del ciudadano promedio; de nuevos enfoques, como el aprendizaje significativo, en diversas áreas: cognoscitiva (saber), psicomotora (saber hacer, aptitudes); afectiva (saber ser, actitudes y valores)».

Con el objetivo de que el alcance de las competencias laborales sea mayor y eficiente, el Estado costarricense ha implementado la formación por competencias en los colegios técnicos del país, lo que facilita que más individuos las adquieran y logren ser competitivos e insertarse en la fuerza productiva de la nación. La educación formal puede y tiene en la educación no formal, un recurso que le permita llenar los vacíos existentes en el sistema. Torres (2007) afirma que un tipo de educación puede verse reforzado y favorecido por algunos recursos de los otros tipos de educación, en este caso, la administración de la educación no formal. De esta manera, los colegios técnicos que pertenecen a la educación formal tienen en la administración de la educación no formal, un aliado para mejorar y ampliar su oferta educativa y complementar, con las modalidades y espacios que utiliza ella, la visión de los integrantes de las organizaciones educativas formales. 
Desde el enfoque de educación no formal, por su plasticidad, se pueden desarrollar diferentes métodos o formas para dar seguimiento a los procesos que se llevan a cabo en los colegios técnicos, en función de la implementación de la evaluación por competencias. El presente proyecto busca hacer un diagnóstico de la evaluación por competencias en el Colegio Técnico Profesional de San Sebastián (CTPSS); de esta manera, se han planteado los siguientes objetivos:

\section{Objetivos}

- Analizar el programa "Evaluación por Competencias" implementado por el MEP en la Educación Técnica en el CTPSS, desde su ejecución.

- Diagnosticar el conocimiento que tienen los educadores del CTPSS en cuanto a la evaluación por competencias.

- Identificar las dificultades delos docentes en la aplicación dela evaluación porcompetencias emanada por MEP.

- Reconocer las ventajas de parte de los docentes en la aplicación de la evaluación por competencias emanada por el MEP.

El Colegio Técnico Profesional de San Sebastián, es un centro de educación secundaria, perteneciente a la Dirección Regional 1 de la provincia de San José. Este centro educativo posee una matrícula de 718 estudiantes y cuenta con un personal docente de, aproximadamente, 56 funcionarios. Este centro educativo, desde hace aproximadamente 4 años, lleva a cabo la implementación del Programa de Competencias emanado por el MEP, el cual sustenta el desarrollo de esta investigación.

\section{Lineamientos conceptuales del Programa de Evaluación por Competencias del MEP}

Según la directriz del Consejo Superior de Educación (SE-339-2003)², se menciona comoúnicoeje transversal del currículo costarricense el de valores, el cual engloba los siguientes temas transversales:

- Cultura ambiental para el desarrollo sostenible,

- Educación Integral de la Sexualidad,

- Educación para la Salud

- Vivencia de los Derechos Humanos para la democracia y la Paz.

Para estos temas, se definió una serie de competencias para el desarrollo de la formación estudiantil. Entendiendo las competencias, según el MEP (2002, p. 1), mediante la Comisión

2 Ministerio de Educación Pública, 2002. La transversalidad en los Programas de Estudio. 
Nacional Ampliada de Transversalidad, como "un conjunto integrado de conocimientos, procedimientos, actitudes y valores, que permiten un desempeño satisfactorio y autónomo ante situaciones concretas de la vida personal y social". Las mismas deben orientar los procesos educativos y el desarrollo mismo de la transversalidad.

Se define como competencias de la transversalidad "aquellas que atraviesan e impregnan horizontal y verticalmente, todas las asignaturas del currículo y requieren para su desarrollo del aporte integrado y coordinado de las diferentes disciplinas de estudio, así como de una acción pedagógica conjunta" (MEP, 2002, p. 2). Seguidamente, se indica cada tema transversal y su competencia por desarrollar, según el MEP:

\section{Tabla 1}

Tema transversal y su competencia

\begin{tabular}{|c|c|}
\hline Tema transversal & Competencia \\
\hline $\begin{array}{l}\text { Cultura Ambiental para el Desarrollo } \\
\text { Sostenible }\end{array}$ & $\begin{array}{l}\text { Aplicar conocimientos críticos y reflexivos de la } \\
\text { realidad social y ambiental. }\end{array}$ \\
\hline Educación Integral de la Sexualidad & $\begin{array}{l}\text { Valerse de principios provistos de valores, } \\
\text { principios éticos, morales sobre la vida, el amor, } \\
\text { la familia y la convivencia }\end{array}$ \\
\hline Educación para la Salud & $\begin{array}{l}\text { Vivenciar un estilo de vida que permita al } \\
\text { individuo mantener y mejorar la salud integral y } \\
\text { la calidad de vida propia y la de los demás. }\end{array}$ \\
\hline $\begin{array}{l}\text { Vivencia de los Derechos Humanos para la } \\
\text { Democracia y la Paz }\end{array}$ & $\begin{array}{l}\text { Pone en práctica los principios básicos de } \\
\text { los seres humanos, tanto para él como a las } \\
\text { personas que lo rodean. }\end{array}$ \\
\hline
\end{tabular}

Nota: MEP, 2002.

Los docentes deben partir de una lectura exhaustiva de los conocimientos previos del estudiantadoy su contexto; enfunción deello, elegir los objetivos de los programas que representan oportunidades para abordar la transversalidad y para el desarrollo de las competencias.

\section{Orientaciones generales para la labor docente}

La evaluación de las competencias son evidencias evaluables; son productos observables y medibles que se esperan del estudiantado. La consecución de éstos, permitirá al docente dar seguimiento al avance individual de cada educando y realimentar el transcurso de aprendizaje, cuando 
así lo requiera el alumno. Los criterios para la evaluación de las competencias son la base para elaborar pruebas teóricas o de ejecución, ya que en ellas se refleja el producto final esperado en cada objetivo.

En el marco de referencia conformado por el Modelo de Educación basada en Normas de Competencia del MEP, el proceso deenseñanza-aprendizaje proporciona conocimientos, desarrolla habilidades y destrezas, para lograr cambios en las actitudes y aptitudes del estudiantado.

\section{Referente teórico}

\section{Educación No Formal, conceptualización.}

La educación no formal es un método usado tanto en el área social como educativa, en los cuales se diseñan, ejecutan y evalúan acciones desde estos dos elementos, con el objetivo y meta de corregir y optimizar las circunstancias de vida de diversos grupos sociales, a través de ordenamientos inclusivos impulsores de cambios sociales e individuales.

Según Colom, Sarramona y Vásquez (1998, p. 12), la educación se concibe como "un proceso permanente vinculado a la mejora de las condiciones de vida de los individuos y las comunidades". Asimismo, Torres (2007) concibe la educación como "acción compleja y heterogénea que se caracteriza por una amplia variedad de procesos, agentes o instituciones" (p. 9). En consecuencia, las organizaciones o ecosistemas de formación se han ido formando más allá de un sistema escolar que permita responder a necesidades educativas emergentes que requieren mayor flexibilidad en su abordaje. Este autor, enmarca el término educación desde los diferentes tipos de educaciones que existen y que las hace, particularmente, diferentes una de otra. Para este estudio, se debe rescatar la definición de educación no formal como compleja, flexible y con una amplia diversidad de procesos agentes e instituciones, mencionado en el anterior párrafo y que le dan un carácter de continuidad a la educación.

Por otra parte, Rogers (2004) considera a la educación no formal como procesos flexibles, positivos, que pueden acceder a los recursos que estén a su alcance, con el objetivo de mejorar la calidad de vida de los individuos inmersos en los diferentes procesos educativos y que pueden hacer uso de los diferentes tipos de educación que existen. En síntesis, estos procesos sean de educación formal, informal o no formal, pueden ser complementarios entre sí, tomando las características de cada enfoque para mejorar los procesos educativos desde sus diferentes contextos.

A su vez, Luján (2010) conceptualiza a la educación no formal como:

Disciplina aplicada al campo de los procesos socioeducativos; su ámbito de acción implica diseñar, organizar, ejecutar y evaluar actividades educativas con el propósito de 
mejorar las condiciones de vida de diversos grupos humanos, mediante procedimientos participativos promotores de transformaciones individuales y colectivas. (p. 101)

Esto implica, que es toda actividad educativa empeñada en mejorar los procesos educativos culturales y sociales, en pos de mejorar el talante humano y por lo tanto, a la sociedad en sí, construyendo ciudadanos actores de un cambio social, en beneficio de un todo.

\section{La administración de la educación no formal}

Para conceptualizar la administración de la educación no formal, Lujan (2010), se refiere a ella desde dos dimensiones fundamentales:

Una relacionada con la administración en general, que incluye la formulación, el diseño, la organización, la ejecución, el monitoreo y la evaluación de los planes, programas y proyectos, según sus actividades y tareas; la otra donde predominan los procesos de enseñanza y aprendizaje inherentes a las acciones de esa modalidad educativa. (p. 101)

Además, considera que los nuevos contextos socioculturales y económicos complejos exigen un compromiso con poblaciones de diversas regiones geográficas y sectores económicos; también afirma que los centros de primaria y secundaria consisten en la educación formal, la cual tiene en la educación no formal un aliado para mejorar y ampliar su oferta educativa, lo que le permite complementar con las modalidades y espacios que utiliza la educación no formal, la visión de los integrantes de las organizaciones educativas formales. En concordancia con lo anterior, Torres (2007) afirma que un tipo de educación puede verse reforzado y favorecido por algunos de estos tipos de educación.

La administración de la educación no formal es un mecanismo que articula las posibilidades y espacios de las acciones educativas no formales, que abarcan desde los salones de clase en los centros educativos hasta los lugares de trabajo en las empresas de todos los sectores económicos (Luján, 2010). De manera que, en toda acción educativa no formal, hay una intencionalidad previa que obedece a necesidades, expectativas, intereses, metas y objetivos de los grupos sociales con quienes proyecta su accionar y con base en estos requerimientos, se definen objetivos y metas de desarrollo humano, social y económico. Para esto, es necesaria la planificación, ya que pone en perspectiva, de manera sistemática, flexible y abierta a cambios, durante y en el transcurso de la planificación, los acontecimientos y las acciones para impactar en el futuro y obtener beneficios esperados con los recursos disponibles en un contexto socioeconómico y cultural determinado.

La administración de la educación no formal puntualiza en muchos aspectos, ya que intenta organizar y sistematizar una serie de procesos que se dan en la sociedad, los cuales 
antes se encontraban dispersos y que no podían ser evaluados, analizados, diagnosticados ni tomados en cuenta como educación. Sin embargo, para delimitar los alcances y objetivos de este estudio, se debe hacer énfasis en la planificación que posibilita la definición de los planes, programas y proyectos por realizar en determinado tiempo y espacio; la organización es un procedimiento continuo para acomodar y facilitar los recursos obtenidos y adaptarlos a las circunstancias propias de las acciones educativas no formales y del contexto donde se ejecutan, con el propósito de cumplir con los objetivos y metas previamente establecidos.

Además de puntualizar en los diagnósticos de necesidades de capacitación en áreas como computación, idiomas, hotelería, educación, entre otros, que permiten obtener datos e información pertinentes para diseñar procesos socioeducativos. En síntesis, se debe entender la administración de la educación no formal como todo tipo de educación fuera de los resguardos y límites de la educación reglada y escolariza, que planifica un conjunto de acciones y actividades que tienen el propósito de influir en procesos educativos para alcanzar una situación elegida como deseable, con los recursos disponibles.

\section{Competencias, conceptualización}

El concepto de competencias laborales es amplio. Al respecto, Villa y Poblete, citados en Martínez, Martínezy Muñoz (2008), mencionan que esta modalidad formativa o enfoque educativo es una manera de responder a las necesidades personales, sociales, profesionales y culturales que plantea la sociedad, en la actualidad. Con este nuevo enfoque de formación, se pretende una evolución en el enfoque tradicional, es decir, de una formación centrada en el aprendizaje.

Para comprender la importancia de la formación basada en competencias y la transformación que representa en el proceso de enseñanza-aprendizaje, se hace necesario establecer comparaciones con respecto al enfoque de formación tradicional de educación, tal y como se detalla en la siguiente tabla:

\section{Tabla 2}

Formación basada en competencias y formación tradicional de educación

\begin{tabular}{ll}
\hline \multicolumn{1}{c}{ FORMACIÓN TRADICIONAL } & \multicolumn{1}{c}{ FORMACIÓN BASADA EN COMPETENCIAS } \\
\hline Desconectada de la realidad del entorno & $\begin{array}{l}\text { Atenta a las necesidades cambiantes de la sociedad } \\
\text { y sus profesionales. } \\
\text { Enfoque centrado en la enseñanza. }\end{array}$ \\
$\begin{array}{l}\text { Enfoque centrado en el aprendizaje y la gestión } \\
\text { del conocimiento. }\end{array}$ \\
Prima la transferencia de información. & Importancia dela formación integraly permanente.
\end{tabular}


Desconocimiento de los intereses de los Se parte de la necesidad de potenciar las estudiantes y de la necesidad de potenciar sus capacidades y habilidades. competencias genéricas, transversales y específicas de los colectivos a los que va dirigida.

Currículo comportamentalizado y poco flexible. Clase magistral como metodología única. Currículo integrado y flexible.

Alumno receptor pasivo de información. Metodología diversa, activa y participativa. Uso de texto escrito como prioritario. Alumno agente de su propio aprendizaje. Centrada en otras formas alternativas de trabajo. Nota: (Martínez et al., 2008).

En el enfoque de formación basado por competencias (FBC), el alumno (aprendiz, en este enfoque) es el centro del proceso de enseñanza-aprendizaje; lo más importante es lo que él tiene que aprender. El objetivo de la FBC es que el aprendiz logre un aprendizaje complejo, que integre el saber, el saber hacer, el saber ser y el saber estar.

El diseño del currículo se inicia con la identificación delas competencias queel mundo profesional/ laboral demanda, las cuales integran el perfil profesional del egresado, ya que éste comprende el conjunto de competencias esenciales que el estudiante debe haber adquirido al terminar sus estudios, para hacer frente a las necesidades de la sociedad, de la profesión y del campo laboral.

El profesional del siglo XXI, debe de ser un profesional competente en los aspectos conceptuales y técnicos de su profesión y, además, poseer un conjunto de características personales (competencias participativas y personales) que son esenciales para alcanzar un desempeño superior ante los desafíos que plantea la realidad actual.

La educación tradicional está frecuentemente orientada, de forma exclusiva, al logro de algunas de las competencias específicas, dejando de lado las competencias genéricas, que son las que hacen del profesional no sólo un experto en su campo, sino un profesional de excelencia.

La formación basada en competencias permite que se establezca una relación directa entre las competencias requeridas y los contenidos de la formación; de esta forma, quienes llevan a cabo el proceso, tendrán un referente para adecuar sus programas formativos y quienes demanden sus servicios, tendrán la seguridad de que se adapten a sus necesidades (Valverde, citado en Martínez et al., 2008).

Para el conocimiento y evaluación por competencias, la definición conceptual que se maneja, de acuerdo con Pulgar (2005) es que:

(...) el conocimiento se logra a través del estudio o la práctica. Se alcanzan habilidades y comportamientos de manera eficiente e instrumental, debidoal refuerzo yel examen de modelos sociales que se transforman en referencias del que asimila. Intervienen factores cognitivos. (p. 18) 
Asimismo, Piaget, citado por Pulgar (2005), considera que "lo que se puede aprender en cada momento depende de la propia capacidad cognitiva, de los conocimientos previos y de las interacciones que se puedan hacer con el medio" (p. 18). Enmarcando, de esta forma, el concepto de conocimiento que referencia este estudio.

Segura (2009, p. 1), por su parte, entiende la evaluación de los aprendizajes como“un proceso constante de producción de información para la toma de decisiones, sobre la mejora de la calidad de la educación en un contexto humano social, mediante sus funciones diagnóstica, formativa y sumativa". Además, señala que "la evaluación es la que mide cuantitativa y cualitativamente los conocimientos y habilidades que se poseen" (Segura, 2009, p. 19). En consecuencia, la evaluación es una herramienta fundamental de medición para la evaluación de los aprendizajes; en este caso, se hace estratégica para los programas educativos que permitan conocer, con criterios válidos, los alcances de los aprendizajes, en función de los objetivos, metodologías y programas planteados.

Con base en lo anterior, la competencia también es considerada por Gonczi y Athanasou, citados por Rojas (2000), como una estructura compleja de atributos generales (conocimientos, actitudes, valores y habilidades), requeridos para interpretar situaciones específicas y desempeñarse en ellas, de manera inteligente. Llama la atención en este planteamiento, la complejidad que implica este tipo de procesos para el adecuado desempeño de los individuos, en los procesos de formación y adquisición de los aprendizajes.

Según el MEP (2002, p. 1), las competencias son consideradas como "un conjunto integrado de conocimientos, procedimientos, actitudes y valores, que permiten un desempeño satisfactorio y autónomo ante situaciones concretas de la vida personal y social". Las mismas deben orientar los procesos educativos y el desarrollo mismo de la transversalidad, con el propósito de alcanzar una mejor calidad educativa en los distintos programas educativos que emplean este enfoque.

Las competencias son un proceso indeleble de elaboración y observación de información que reflexiona en un modelo, en relación con criterios que propician en el estudiantado, la realimentación para continuar obteniendo aprendizaje cuando evidencia sus capacidades metacognoscitivas en contextos reales, con una interacción permanente entre instancias educativas implicadas (Segura, 2009). La noción de competencia implica la habilidad para hacer algo o la capacidad para llevar a cabo una tarea.

Por su parte, Mora citado en Segura (2009), considera que una evaluación por competencias demanda un diseño del método de estudios elaborado por competencias. Este planteamiento está acorde con la implementación del enfoque que, por lo general, no siempre se da en los programas educativos que lo emplean. Esta situación, en algunos casos, provoca contradicciones entre el currículum y el enfoque de la evaluación por competencias, desde su manera de evaluar, al no coincidir con la evaluación de los aprendizajes establecida. 
La evaluación por competencias es un conjunto de destrezas y habilidades que el individuo, en la actualidad, debe poseer y saber, para poner en práctica en su contexto educativo; de esta forma, el individuo cumple con los niveles de desempeño requeridos. Así, el individuo, la compañía y la sociedad forman una sinergia en la que los actores implicados, se ven beneficiados de los diferentes procesos productivos y de servicios que la sociedad requiere (Rojas, 2000; MEP, 2002; Pulgar, 2005 y Segura, 2009).

Dentro de la evaluación por competencias, el conocimiento que posee el personal docente que tiene a cargo los distintos programas bajo este enfoque, es fundamental para la eficiente aplicabilidad, por lo cual es indispensable considerar el nivel de asimilación e identificación que tienen los educadores, así como sus principios generales, metodologías y objetivos.

Este conocimiento es en relación con el objetivo que persigue el programa, métodos de evaluación que emplean los profesores, conocimiento del programa, metodología o fuente bajo la cual se adquirió el conocimiento del programa, existencia de instrumentos para evaluar por competencias, metodología para evaluar por competencias, evaluación del factor central del modelo de educación basado en normas de competencia, permite ser observable, la competencia es inferida, pruebas escritas y orales parte del método de evaluación por competencias, observación del rendimiento parte de la evaluación por competencias.

En consecuencia, es necesario en la evaluación por competencias, tomar en cuenta: resultados con respecto al programa de evaluación por competencias a los objetivos, los criterios de evaluación, las actividades, las técnicas, los instrumentos, el conocimiento de la gestión u operacionalidad, la conceptualización, los procesos educativos en general que se llevan a cabo, las necesidades, actividades, instrumentos, técnicas y actividades metodológicas que ofrece el enfoque para guiar al profesor en su aplicación.

En la evaluación de los programas por competencias, el nivel de dificultad que enfrentan los docentes es un elemento fundamental a considerar, tomando en cuenta que, de una adecuada ejecución dependerá la calidad del proceso de enseñanza y aprendizaje, en gran medida, razón por la cual es relevante conocer las limitaciones y obstáculos en los aprendizajes asociados a los procesos requeridos para la asimilación de la evaluación por competencias, por parte de los actores que forman parte del proceso, ya que es fundamental en su ejecución para que los estudiantes a su vez, asimilen una serie de conocimientos, procedimientos, actitudes y valores que le permitan un desempeño satisfactorio y autónomo ante situaciones concretas de la vida personal y social.

Entre las principales limitaciones que se pueden encontrar en la ejecución de los programas de evaluación por competencias y tomando en cuenta los lineamientos del MEP (2002), están: desempeño en forma adecuada, conocimiento de los profesores de los objetivos, juicio adecuado 
de la metodología empleada, dificultades de aplicación del enfoque, desconocimiento de los lineamientos del programa, resultados y registros, para dar seguimiento al proceso y dominio de la información teórica de la evaluación por competencias.

Como elementos que favorecen la aplicación del programa de evaluación por competencias en los docentes, también se consideran los conocimientos, procedimientos, actitudes y valores, en busca de un adecuado desempeño (MEP, 2002).

\section{Referente metodológico}

\section{Tipo de investigación.}

El estudio se realizó mediante la investigación cualitativa, con un enfoque descriptivo. Al respecto, según Hernández, Fernández y Baptista (2008, p. 8), “la investigación cualitativa utiliza la recolección de datos sin medición numérica para descubrir o afinar preguntas de investigación en el proceso de interpretación". En este caso, se diagnostica en cuanto al conocimiento que tienen los educadores del CTPSS en la evaluación por competencias emanada por el MEP, la identificación de las dificultades que viven los docentes en la aplicación de la evaluación por competencias emanada por el MEP, el reconocimiento de las ventajas de parte de los docentes en la aplicación de la evaluación por competencias por parte del MEP.

Según Hernández et al. (2008), la investigación descriptiva "busca especificar propiedades, características y rasgos importantes de cualquier fenómeno que se analice. Describe tendencias de un grupo o población" (p. 102). En este estudio, se describe el programa de "Evaluación por Competencias" implementado por el MEP y su impacto en los actores implicados en su ejecución.

Se pretende, mediante este paradigma cualitativo, enfoque descriptivo, conocer la experiencia docentes y de los directivos sujetos del estudio, sobre la implementación del Programa por Competencias, para lo que se llevarán a cabo entrevistas, con el fin de realizar un análisis, con un margen aceptable de confiabilidad, verificar los obstáculos, ventajas y desventajas de los docentes al trabajar en la aplicación de la evaluación por competencias.

La recolección de la información se sustenta en una entrevista con 15 tópicos, mediante preguntas abiertas y cerradas, en relación con: los criterios de evaluación, las actividades, las técnicas, los instrumentos, el conocimiento de la gestión, la operacionalidad, la conceptualización y el proceso educativo, en general. 


\section{Análisis de resultados}

\section{Diagnóstico del conocimiento de los docentes acerca del enfoque de evaluación por competencias.}

Siendo el conocimiento "una capacidad que se adquiere por medio del estudio o la experiencia y en el cual se adquieren destrezas y conductas de modo operante e instrumental" (Pulgar, 2005, p. 19) y con el propósito de conocer el conocimiento del personal docente del Colegio Técnico San Sebastián sobre el enfoque Evaluación por Competencias, el cual consiste en un "cúmulo integrado de conocimientos, procedimientos, actitudes y valores, que potencie y motive al estudiante a un desempeño satisfactorio y autónomo ante situaciones concretas de la vida social y personal" (MEP, 2002, p. 1), se procedió a consultarle a los docentes, al coordinador técnico y a la directora de la institución su conocimiento al respecto de la implementación de éste en la educación costarricense, a partir de los siguientes indicadores: criterios de evaluación, claridad conceptual del programa, actividades metodológicas, técnicas, instrumentos, conocimiento de la gestión u operacionalidad, conceptualización y proceso educativo en general que se lleva a cabo.

Con respecto al conocimiento que tienen los educadores del CTPSS, en cuanto a los criterios de evaluación del enfoque por competencias emanada por el MEP, la mayoría de los entrevistados dice manejar un concepto claro de lo que es la evaluación por competencias, mientras que una minoría del profesorado o administradores institucionales encargados de la gestión, no lo consideran así; justifican que lo confunden entre los dos tipos de evaluación, la evaluación por competencias y el Reglamento de Evaluación de los Aprendizajes (REA). Lo anterior, debido a que las capacitaciones e informaciones que los profesores han recibido por parte del MEP son escuetas, cortas, con poco conocimiento de los capacitadores, lo que conlleva a "contradicciones entre el discurso y la práctica" Segura (2009); además, este autor agrega que "el currículo costarricense (...) debería de haber cambiado de la comprobación de logros del estudiantado, a la promoción de competencias, para poder cumplir con los mandatos de la política educativa vigente,(...),se debe ampliar la noción de los aprendizajes del MEP" (p. 3). En síntesis, el desconocimiento de los profesores se da, no en relación con el concepto de Evaluación por Competencias, sino de todo lo que implica este modelo evaluador; dado que todo el currículo costarricense se debe de modificar para que no se den contradicciones y no se dé el enfoque de manera incompleta, con mezcla de otro currículo.

Por ejemplo, uno de los entrevistados afirma que "no hay ningún criterio de evaluación por competencias, únicamente se cuenta con un programa por competencias con evaluación sumativa, que consiste en dos exámenes de $20 \%$ cada uno". Sin embargo, el concepto de evaluación por 
competencias parece estar claro; aunque en su mayoría, existe una opinión generalizada, por parte de los profesores, que el conocimiento que tienen de la evaluación por competencias es muy poco; solo algunos profesores lo conocen a profundidad. Otros docentes alegan que han recibido capacitaciones, públicas o privadas, con respecto al enfoque y aún no lo manejan. Esto evidencia que existe una política para la divulgación del enfoque que parece darse de forma lenta pero progresiva; no obstante, su orden y aplicación no son del todo coherentes y organizadas.

Además de cuestionar a los profesores con respecto a su conocimiento, se realizaron preguntas acerca de la comprensión y uso de las diferentes herramientas metodológicas que, desde el MEP, se ofrecen para la implementación del enfoque, a lo que la mayoría respondió afirmativamente, por cuanto manifiestan estar al tanto de la existencia de instrumentos para la aplicación del enfoque; pocos consideran que no existe ningún tipo de enfoque.

Lo anterior, evidencia el contexto que rodea el enfoque fuera y dentro del colegio, ya que si existen instrumentos pero no en todas las áreas, como lo afirma la directora: "Bueno en el área académica no se evalúa por competencias, y en el área técnica hay una parte la otra es también académica, no es totalmente una evaluación por competencias". También se considera que la administración del MEP o del colegio, no brinda la información de manera adecuada o el profesor no lee los programas completos. Esto se hace más evidente en la diferencia de criterios bajo la no contestación de la operacionalidad del cómo evaluar usando el enfoque; si bien es cierto, el enfoque brinda posibilidades, lo que busca es estandarizar, de alguna manera, la forma de evaluar.

Con el objetivo de verificar lo mencionado por los profesores con respecto al conocimiento y manejo del enfoque por competencias, se les hizo una serie de preguntas para percibir la concordancia de criterios con respecto a los conceptos del enfoque, según el contenido programático del plan brindado por el MEP y la identificación de los profesores con respecto a estos conceptos. Los hallazgos arrojan que los docentes conocen algunos de los conceptos, pero la mayoría de ellos no están claros.

En síntesis, con respecto al conocimiento de los educadores del CTPSS en cuanto a la evaluación por competencias emanada por el MEP, éste es difuso debido a varios factores: la mala comunicación entre entes administrativos, dentro y fuera del colegio, la no claridad del enfoque brindado por el MEP y el rol paciente tomado por los profesores al dejarse ordenar una implantación de forma tan desorganizada. Es el sentir de varios investigadores, que la implantación de un currículo basado en competencias, desde el MEP, debe abarcar el estudio de la interdisciplinariedad que se manifiesta en el saber (ser, convivir, hacer y conocer). Por ejemplo, Segura (2009) afirma:

La modalidad de evaluación que debe de ponerse en práctica por tanto, cuando de lo que se trata es de evaluar el logro de los aprendizajes de cada alumno en función de criterios 
de logro previamente establecidos. Para ello es necesario que estos criterios de evaluación estén formulados de modo concreto y claro, ya que lo que se pretende es que el alumno alcance un determinado nivel en una determinada materia o que aprenda hacer algo previamente fijado, intentando establecer la armonía entre los resultados conseguidos y los objetivos propuestos. (p. 30)

Al respecto, es importante considerar que, siendo la administración de la educación una disciplina encargada de velar por la sana conducción de las organizaciones educativas, tal y como lo afirma Lujan (2010, p. 107), "la administración incluye una compleja gama de fases determinadas por la planificación, la organización, el monitoreo y la evaluación de las actividades humanas que permiten brindar servicios y la producción de bienes de manera eficiente y eficaz", no pareciera que, en este caso específico, la gestión de la educación asuma el papel que le corresponde; es importante corregir estos vacíos y poder, de esta forma, alcanzar los objetivos educativos propuestos con respecto a este programa.

\section{Identificar las dificultades de los docentes en la aplicación de la evaluación por competencias emanada por el MEP.}

Para esta investigación, se define como dificultades, las limitaciones y obstáculos en los aprendizajes asociados a los procesos requeridos para la asimilación de la Evaluación por Competencias de los actores que forman parte del proceso que el MEP está implementando. En específico, estas limitaciones de los docentes están ligadas al "conjunto integrado de conocimientos, procedimientos, actitudes y valores, que permite un desempeño satisfactorio y autónomo ante situaciones concretas de la vida personal y social" (MEP, 2002, p.1). Las preguntas realizadas a los sujetos participantes, se desarrollaron con base en los siguientes indicadores: posibilidades para desempeñarse en forma adecuada, conocimiento de los profesores de los objetivos del programa Evaluación por Competencias del MEP, dificultad de aplicación del enfoque por diferentes entes organizacionales del colegio y el MEP, juicio(s) adecuado(s) de la metodología empleada en el programa de Evaluación por Competencias para la evaluación estudiantil, desconocimiento de los lineamientos del programa/contradicciones/realidad educativa/disponibilidad hacia los cambios, resultados y registros, para dar seguimiento al proceso/dominio de la evaluación teórica del enfoque, utilización de los instrumentos metodológicos del enfoque.

Concerniente a las dificultades que tienen los docentes en la aplicación de la Evaluación por Competencias, hay una marcada mayoría que menciona la poca claridad de las directrices; otro número importante de profesores afirma que una de las dificultades es la falta de capacitación por parte del MEP, lo que imposibilita a su desempeño de forma adecuada, ya que su conocimiento no es claro acerca de los objetivos y sus juicio(s) están condicionados a una metodología que no tienen clara, como hacen constatar los indicadores. Un ejemplo de 
esta situación, es cuando uno de las personas entrevistadas afirma que: "Las capacitaciones que dan ni ellos mismos saben en qué consisten". Otra respuesta relacionada con la aplicación del programa, indica que: "No queda claro su aplicación si los estudiantes pasan por el REA".

También, concerniente a las dificultades, se consultó si el enfoque favorece u obstaculiza la evaluación académica al estudiante y sus principales limitaciones. Una importante mayoría dijo que no favorecía el juicio que se le debía aplicar a los estudiantes; una minoría respondió afirmativamente; mientras que otros participantes continúan mencionando la poca claridad en el enfoque y las altas cargas de trabajo al profesor.

Además, mencionan como limitaciones, nuevamente, el desconocimiento y una minoría indica que la descontextualización del enfoque; un ejemplo de estos comentarios lo brindan las dos siguientes afirmaciones, dadas por el coordinador técnico y un profesor, respectivamente: "Ni una ni otra pues evaluación por competencias no ha madurado lo suficiente"; el profesor menciono: "Inexperiencia en el enfoque, faltante de recursos, poca capacidad o creatividad para planear".

Las afirmaciones que, desde la mayoría de los profesores se perciben, es que la Evaluación por Competencias es una directriz o política mal implementada para cumplir con la agenda, tal como lo afirma Morales, citado en Segura (2009), al considerar que "la evaluación por competencias empezó como un proyecto piloto en el año 2000, que tuvo que adecuarse a la normativa vigente"(p. 133), mismo que, forma gradual, se ha ido incorporando en los programas de estudio, según especialidades de los colegios técnicos profesionales, lo que ha generado algunas contradicciones entre el enfoque y lo propuesto en el REA. Lo anterior, sin tomar en cuenta a los principales actores del proceso educativo, quienes lo miran, en algunos casos, como una carga más de trabajo que no funciona en el contexto costarricense.

\section{Reconocer las ventajas de parte de los docentes en la aplicación de la Evaluación por Competencias emanada por el MEP.}

Para la investigación, se entiende por ventajas de la evaluación por competencias, a los elementos que favorecen la aplicación del programa por parte de los docentes, quienes buscan desarrollar en el alumnado un "currículo integrado de conocimientos, procedimientos, actitudes y valores, que potencie y motive al estudiante a un desempeño autónomo ante situaciones concretas de la vida social y personal" (MEP, 2002, p. 1). Los indicadores utilizados para conocer las ventajas de la implementación del programa son: ventajas de aplicación del enfoque por diferentes entes organizacionales del colegio y del MEP, ventajas del enfoque en la evaluación del estudiante y ventajas que experimenta el profesorado para emplear el enfoque Evaluación por Competencias.

En relación con las ventajas que perciben los docentes en la aplicación de la evaluación por competencias del MEP, existe una pequeña mayoría que percibe ventajas significativas, mientras 
que una minoría no ve ningún aspecto positivo. Es significativo que una cantidad importante de educadores afirma que el apoyo que se da entre compañeros es una ventaja a tomar en cuenta dentro del enfoque. Entre los comentarios positivos, una de las personas entrevistadas afirma que: "busca integrarnos y hacernos más competitivos en un mundo globalizado".

La diferencia de criterios es muy marcada, dado que los profesores saben que esto es un enfoque mundial y tiene sus aspectos positivos, pero se encuentran divididos en cuanto a qué tan positivo es para el contexto nacional, lo que provoca resistencia entre los profesores.

A las preguntas relacionadas con las ventajas del enfoque en la evaluación académica al estudiante y las ventajas que perciben los profesores para emplear este enfoque, las respuestas refuerzan, en una pequeña mayoría, que se perciben mejoras, aunque la minoría indica que no. Se destacan los comentarios de los profesores, quienes afirman que experimentan más carga de trabajo, pero que permite una evaluación más real a lo que los estudiantes se van a encontrar en el mercado laboral. Estos resultados reflejan lo que la teoría resalta y lo mencionado por la directora y algunos profesores. Para ejemplificar, Segura (2009) afirma que: "la Evaluación por Competencias se ha ido introduciendo poco a poco en los programas de estudio de las diversas especialidades de educación técnica (...) y que existen (...) contradicciones con lo propuesto en el REA vigente y el hecho que dichas asignaturas son medidas en pruebas nacionales" (p. 14).

En síntesis, las contradicciones y la falta de políticas coherentes y concretas desde el MEP, causa la resistencia de los profesores a la evaluación por competencias, ya que no existen directrices claras, lo que provoca en los profesores un desgaste de tiempo, fuerza y energía en una disposición que no ha sido modificada para emplearse, de una manera más adecuada, al entorno nacional. Al respecto, Segura (2009) afirma que:

(...) desde la evaluación de los aprendizajes, no han existido políticas claras del MEP, acerca de cómo evaluar las competencias desde el centro educativo y en las actividades de mediación pedagógicas, esto se debe a la necesidad de consensuar un Currículo Nacional Básico que articule la evaluación con el currículum para que no existan contradicciones. (p. 17)

Lo anterior, sin tomar en cuenta los temas de recursos e infraestructura que además, cuestionan los profesores.

La información recopilada sugiere que, superficialmente, la mayoría de los educadores tiene el conocimiento de lo que es evaluación por competencias. Sin embargo, cuando se trata de la operacionalidad y el manejo a profundidad de los criterios de evaluación, conceptos del programa, actividades metodológicas, técnicas, instrumentos, conceptualización y el proceso educativo, existen discrepancias por las contradicciones y deficiencias mencionadas, en entorno a la aplicación del programa en estudio. Es importante indicar que esto no aplica a todas las áreas y a todos los profesores, debido a que la implementación es progresiva y el conocimiento 
de la aplicación de la evaluación por competencias está supeditado al manejo que se le da por parte de los diferentes actores implicados en la gestión, desde la educación no formal e implementación de este enfoque.

Las dificultades mencionadas por los docentes, en cuanto a la evaluación, resaltan la poca claridad de las directrices y la falta de capacitación, lo que causa un desempeño inadecuado por la poca información de los objetivos y su aplicación, limitando su juicio a una metodología que no maneja el profesorado. Además, de la descontextualización de la metodología, ya que es importada y debe contextualizarse de manera más acorde con las capacidades del sistema educativo costarricense. Al respecto, se evidencia la necesidad de fortalecer los procesos de la administración de la educación no formal, en la implementación de este enfoque.

Los profesores y profesoras reconocen ventajas del enfoque evaluación por competencias. Sin embargo, existe resistencia por parte de estos a su aplicación. Se rescatan algunas ventajas de aplicación, en cuanto a que beneficia la cohesión de los educadores, ya que se da intercambio de información para la implementación adecuada del enfoque. También, mencionan ventajas en cuanto a la aplicación de la evaluación académica del estudiante.

Los educadores tienen el conocimiento de que este enfoque es un tema global y afirman que se considera positiva la integración de Costa Rica al mismo. Sin embargo, los profesores mencionan, en este apartado, una clara resistencia, ya que, desde su punto de vista, aumenta su carga de trabajo. Hay que tomar en cuenta que no todos los profesores poseen o han buscado la información para la aplicación del enfoque. En este contexto, desde la gestión de la educación no formal, es posible habilitar espacios de divulgación, inducción y capacitación para que el profesorado adquiera competencias adecuadas que le permitan una implementación correcta, según los objetivos del MEP.

Si bien es cierto la aplicación del modelo de evaluación por competencias posee contradicciones entre el discurso y la práctica, este enfoque es una herramienta flexible, la cual puede ser modificada e implementada por los docentes en el momento de su cotidianidad, de acuerdo con la pertinencia en su quehacer, ya que el programa incentiva que los elementos posean íntima relación con los objetivos propuestos en el plan de estudios entregados por el MEP. De esta manera, docente y dicente pueden compartir puntos de vista acerca de un tema de clases y ligarlo al tejido de lo que las personas, familias, comunidades, sociedades y países viven en la aldea global y así, construir una sociedad y un mundo más sano, diverso e inclusivo, aprendizaje para la vida.

El análisis señala como ventaja, la cohesión entre el grupo de docentes al socializar diferentes metodologías para la correcta implementación del enfoque. Según Castellanos, citado por MEP (2002), el único eje transversal del currículo en el país, es el de valores, el cual 
"impregna todo el currículo costarricense y sus asignaturas y requieren para su desarrollo del aporte integrado y coordinado de las diferentes disciplinas de estudio, así como una acción pedagógica conjunta" (p. 2).

Lo anterior, se encuentra en estrecha relación con lo mencionado y lo expuesto en los resultados, en cuanto a las ventajas de la evaluación por competencias en el CTPSS, dado que es un factor a incentivar dentro del marco de una capacitación que mejore la implementación de la evaluación por competencias, por ser uno de los puntos fuertes encontrados en esta investigación. Además, se busca estimular esta ventaja, mediante la articulación entre departamentos para mejorar el proceso de implementación de la evaluación por competencias, a través de mecanismos que provean socialización de conocimientos, retroalimentación, sistematización de información; así, los docentes se convierten en actores fundamentales en la implementación e incluso mejora del enfoque, al tener la información más sistematizada y coordinada entre sí.

También, de las ventajas encontradas y que pueden ser mejoradas en la implementación de una capacitación de la administración de la educación no formal, se encuentra la aplicación de la evaluación académica del estudiante. Esta busca ser una evaluación en concordancia a lo que ellos experimentarían en el campo laboral, dado que este enfoque busca ser un aprendizaje para la vida del estudiante, para desarrollarse, de forma autónoma, en contextos laborales y sociales.

Es fundamental que, desde la perspectiva de una capacitación de la administración de la educación no formal, se incentiven las ventajas de este enfoque, el cual tiene como objetivo la formación de ciudadanos cada vez más conscientes de su entorno, para así mejorarlo por medio de los elementos que vienen enraizados en la propuesta del enfoque, como por ejemplo, transparencia, flexibilidad, manejo de problemas, proactividad y destrezas, con el objetivo de insertar al país en el contexto global y por ende, a los estudiantes en el mercado laboral.

Con la asimilación del enfoque por parte de los docentes y el progresivo cambio de paradigma por parte de los diferentes actores encargados de la implementación del enfoque, se logra fundar en el proceso de enseñanza aprendizaje, la noción de que la sociedad se construye juntos y que el proceso de enseñanza y aprendizaje solo puede tener significancia si existe posicionamiento, de aprendices y agentes junto con los dicentes de cambio, a través de relaciones justas, transparentes y horizontales.

Recapitulando, es un rol fundamental de los programas de la administración de la educación no formal, organizar, planear y mejorar programas socio-educativos que antes se encontraban dispersos y no podían ser diagnosticados, sistematizados ni evaluados. Los resultados arrojados en esta investigación, son un incentivo y una reafirmación de que los programas de administración de la educación no formal, como educación paralela a la educación formal, pueden subsanar vacíos en el sistema tradicional. 
Un ejemplo de esto, es cuando uno de los educadores menciona:"Inexperiencia en el enfoque, faltante de recursos, poca capacidad o creatividad para planear". Esta afirmación plantea la necesidad de una capacitación en evaluación por competencias pero también, sugiere una capacitación en planeamiento y creatividad para evaluar por competencias, recalcando la afirmación de que la educación no formal puede trabajar, de forma paralela, al sistema formal, con el fin de aportar espacios que mejoren los procesos educativos, desde una comunidad o país.

\section{Conclusiones}

Con respecto al conocimiento que poseen los docentes sobre el Programa de Evaluación de Competencias, se manifiesta que se conoce en que consiste la evaluación por competencias en forma general. Sin embargo, evidencian desconocimiento en los conceptos básicos de la evaluación por competencias, tales como: objetivos, criterios de evaluación, actividades, técnicas, instrumentos, conocimiento de la gestión u operacionalidad, conceptualización, proceso educativo que se lleva a cabo, actividades, instrumentos, técnicas y actividades metodológicas que ofrece el enfoque para guiar al profesor en su aplicación. Lo anterior, podría inferir que las debilidades en la ejecución de este programa, evidenciadas en el estudio según los docentes consultados, se debe al desconocimiento que poseen en los conceptos básicos del programa de Evaluación por Competencias.

Precisamente esta debilidad es un campo de acción que la administración de la educación no formal puede liderar, con el fin de minimizar los vacíos para la operacionalización del programa.

En relación con las dificultades de los docentes en la aplicación de la evaluación por competencias emanada por el MEP, para desempeñarse en forma adecuada, la investigación concluye la existencia de juicio(s) sobre la metodología empleada en el programa de evaluación por competencias para la evaluación estudiantil, dificultades de aplicación del enfoque por diferentes entes organizacionales del colegio y el MEP, desconocimiento de los lineamientos del programa, contradicciones en los lineamientos dados por el MEP y la realidad educativa, disponibilidad hacia los cambios que el programa requiere y los resultados y registros, para dar seguimiento al proceso, dominio de la información teórica de la evaluación por competencias, utilización e implementación de los instrumentos de evaluación por competencias, resultados o escalas de evaluación para verificar los objetivos.

Las principales ventajas de parte de los docentes en la aplicación de la evaluación por competencias emanada por el MEP, permite evidenciar como elementos positivos los elementos a favor de la evaluación académica del estudiante, desde el enfoque en la evaluación, ya se da le da importancia a la valoración del estudiante desde una realidad más cercana a la que se va a enfrentar en el mundo laboral. Además, se rescata que es una metodología global que favorece la inserción del país, en el contexto mundial. 
Entre los factores que conducen al fracaso, se pueden mencionar las contradicciones del currículo que hace que los profesores no sepan si evaluar por el REA, que es necesario seguir para no recibir una apelación; instrumentos para ciertas áreas, otras no los poseen, aplicación de instrumentos de evaluación que son supuestamente necesarios para la empresa en la que el estudiante se incorpora por ser una institución técnica profesional pero que, en realidad, no son tomados en cuenta, lo que causa exceso de trabajo, burocracia y desgaste docente.

En resumen, se aplican diferentes instrumentos metodológicos que son positivos pero también negativos, cuando no se le ha dado al profesor las herramientas necesarias para evaluar o éste no las busca, en caso de existir; limitando el tiempo que el profesor podría utilizar en una correcta evaluación de los proyectos, el extra-clase que pueden ser instrumentos que ayuden a inferir el manejo de una competencia en el aula.

Entre los aspectos positivos se menciona la integración a la globalización. Sin embargo, los objetivos primordiales que se buscan en el alumnado, no se logran en su totalidad y no necesariamente por la aplicación de la evaluación por competencias. Por ejemplo, se logra, en forma parcial, el objetivo referente a inculcar "actitudes, valores y conocimientos que permitan un desempeño satisfactorio y autónomo ante situaciones concretas de la vida personal y social" (MEP, 2002, p. 1), pero se queda debiendo en los procedimientos y parte del conocimiento. Los primeros se logran por medio de los proyectos y otros instrumentos, dado que los profesores inculcan los valores de responsabilidad y disciplina, aunque algunos trabajos sean mediocres.

Entre los aspectos positivos, se rescata que el alumno está más consciente de su evaluación, de todas las posibilidades que le está ofreciendo el sistema, resultando esto en la no adquisición de las competencias específicas, tan primordiales como se hizo ver en el trabajo.

Se evidencia una evaluación por competencias que no logra sus objetivos, según los propósitos de la directriz, la cual tiene poca credibilidad en los administradores de la institución, los profesores y los alumnos, debido a la forma en que se da su implementación, a sabiendas de sus aspectos positivos. Se encuentra necesario una implementación seria y contextualizada, con directrices claras que faciliten el proceso de enseñanza aprendizaje, con el fin de cumplir con los objetivos de brindar una educación de calidad, sin socavar a ninguno de los principales actores inmersos en el proceso de enseñanza aprendizaje y tal vez, no solo el cumplir con una política país y recibir los beneficios que conllevan una disminución en la deserción de los estudiantes.

Los hallazgos del estudio ratifican la importancia de la administración de la educación no formal gestando procesos socioeducativos, en distintos campos del sistema educativo costarricense, por ser ésta una disciplina que parte del análisis de la realidad, la cual es compleja, diversa y cambiante; estas características la convierten en una herramienta ideal para ejecutar planes, programas y proyectos socioeducativos acordes con el contexto según las demandas emergentes. 


\section{Referencias}

Colom, A. J.; Sarramona, J. y Vásquez, G. (1998). Educación no formal y diferenciada. Barcelona: Ariel.

Hernández, R.; Fernández, C. y Baptista, P. (2008). Metodología de la investigación. México: McGraw-Hill Interamericana.

Luján, M.E. (2010). La Administración de la Educación no formal aplicada a las organizaciones sociales: Aproximaciones teórico prácticas. Revista Educación, 34(1), pp. 101-118. Recuperado en: http//www.latindexucr.ac.cr/educación-34-1-2010/educación-34-1-2010-06.pdf.

Martínez, P.; Martínez, M. y Muñoz, J.M. (2008). Formación basada en competencias en educación sanitaria: Aproximaciones a enfoques y modelos de competencia. RELIEVE, 14(2), pp. 1-23. Recuperado de http://www.uv.es/RELIEVE/v14n2/RELIEVEv14n21.htm.

Ministerio de Educación Pública. (2002). La transversalidad en los Programas de Estudio. Comisión Nacional Ampliada de Transversalidad. San José, Costa Rica: Autor.

Pulgar, J.L. (2005). Evaluación del aprendizaje en educación no formal: recursos prácticos para el profesorado. Madrid: NARCEA, S.A.

Rogers, A. (2004). Non-Formal Education: Flexible Schooling or participatory Education. Hong Kong: University of Hong Kong.

Rojas, I. (2000). La educación basada en normas de competencia (EBNC) como un nuevo modelo de formación profesional en México. Pensamiento Universitario, 19, pp. 45-75. Recuperado de http://es.scribd.com/doc/87137018/EBNC-como-un-nuevo-modelo-de-formacionMexic

Ruiz, M. (julio, 2003). Formación por competencias laborales, un reto para la universidad. Ingenium, 4(8), pp. 81-85.

Segura, M.A. (2009). La evaluación de los aprendizajes basada en el desempeño por competencias. Actualidades Investigativas en Educación, 9, (2), pp. 1-25. Recuperado de http://www. latindex.ucr.ac.cr/aie-2009-2/aie-2009-9-26.pdf 
Torres, C. (2007). La educación no formal y diferenciada: fundamentos didácticos y organizativos. Madrid: Editorial CCS.

Vargas Z., F. (1998). La formación por competencias. En: Ministerio de Salud (1998). Gestión de la capacitación en las organizaciones. Conceptos básicos. Recuperado de http://www.minsa. gob.pe/publicaciones/pdf/capacitacion.pdf 